\title{
An unusual cause of upper gastrointestinal bleeding
}

A 57-year-old man presented to us with melena of 3 days' duration. He had undergone laparoscopic cholecystectomy 1 year prior to presentation. He was hemodynamically stable. An upper gastrointestinal endoscopy was carried out and revealed a Hem-o-lok clip (Weck Closure Systems, Research Triangle Park, North Carolina, USA) eroding into the first part of duodenum ( $\bullet$ Figs. 1 and 2 ).

The Hem-o-lok clip could be seen clearly and there was no other cause for upper gastrointestinal bleeding up to the third part of the duodenum. We tried to manipulate and remove the clip with the help of a biopsy forceps but it was not possible or safe to do so, as it was firmly embedded in the wall of the duodenum. On reviewing the patient's operative notes of laparoscopic cholecystectomy, four Hem-o-lok clips had been left in situ, two each on the cystic duct and cystic artery. The upper gastrointestinal bleeding stopped on conservative treatment and the patient was discharged from hospital well.

A follow-up endoscopy 3 months later revealed a hem-o-lock clip loosely attached to the anterior wall of the first part of the duodenum. It was easily removed with biopsy forceps without any complications. There was no bleeding and the patient remained well.

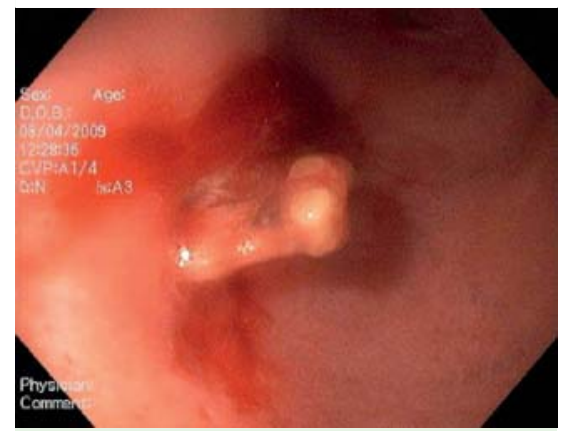

Fig. 1 Closed Hem-o-lok clip seen in the first part of the duodenum.

This is a unique cause of upper gastrointestinal bleeding. Laparoscopic cholecystectomy is a very common operation and Hem-o-lok clips are used quite frequently for clipping the cystic artery and cystic duct. The erosion of Hem-o-lok clips into the duodenum has not been reported in the available literature. Surgeons must be aware of this unique complication, which also needs to be studied further and evaluated in the interest of patient safety.

Competing interests: None

Endoscopy_UCTN_Code_CPL_1AH_2AJ

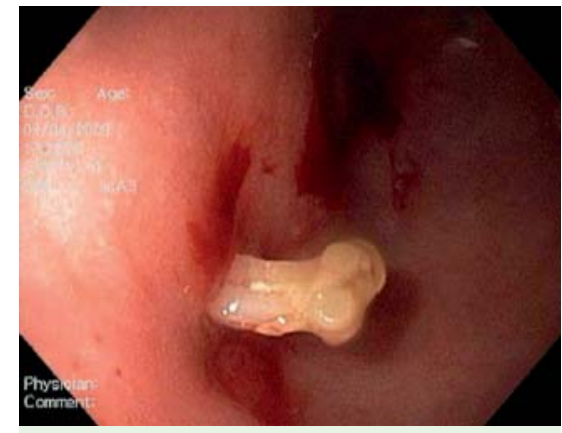

Fig. 2 Closed Hem-o-lok clip seen in the first part of the duodenum (after washing).

\section{S. K. Mantoo ${ }^{1,2}$, J. Rao ${ }^{1}$}

1 Department of Surgery, Tan Tock Seng Hospital, Singapore

2 General Surgery, Alexandra Hospital, Singapore

Bibliography

DOI 10.1055/s-0029-1244010

Endoscopy 2010; 42: E123

(c) Georg Thieme Verlag KG Stuttgart · New York . ISSN 0013-726X

Corresponding author

S. K. Mantoo, MD

General Surgery

Alexandra Hospital

378 Alexandra Road

Singapore 090021

Singapore

Fax: +65-63793540

skmantoo@gmail.com 